TWENTY INNOUATIUE

CONTRIBUTIONS TO THE PEDAGOGICAL STRATEGY

OF THE 2O19 FAU-UNNE GRAPHIC DESIGN

ENTRY COURSE

KEYWORDS

Innovation; incoming student; contributions.

\section{ABSTRACT}

This presentation presents the analysis of the experiences obtained by the group of teaching tutors in the course for entering the Graphic Design career at FAU-UNNE in 2019, proposing an improvement in pedagogical and didactic strategies, detailing twenty contributions grouped into three categories as points of analysis: Teaching methodology, Structuring phases and Consolidation of the student with his new environment, which were beneficial for the incoming students recipients of this proposal, as well as for the teaching practice.
UINTE CONTRIBUIÇÕES

INOUADORAS PARA

A ESTRATÉGIA PEDAGÓGICA

DO CURSO INGRESSO

DE DESIGN GRÁFICO

DA FAU-UNNE 2019

\section{RESUMO}

Inovação; novos participantes; contribuições.

\section{PALAURAS CHAUE}

Esta apresentação apresenta a análise das experiências obtidas pelo grupo de tutores docentes no curso de ingresso na carreira de Design Gráfico da FAU-UNNE em 2019, propondo uma melhoria nas estratégias pedagógicas e didáticas, detalhando vinte contribuições agrupadas em três categorias como pontos de análise: Metodologia de ensino, Fases estruturantes e Consolidação do estudante para com seu novo entorno, que foram benéficas para os destinatários da referida proposta, bem como para a prática docente 


\title{
UEINTE APORTES INNOUADORES A LA ESTRATEGIA PEDAGÓGICA DEL CURSILLO DE INGRESO DE DISEÑO GRÁFICO 2019 DE LA FAU-UNNE
}

\author{
Damián E. AQUINO; Fernando G. ESTÉVEZ; Marisa \\ S. PETTINICHI; Cintia M. SLOBODIUK; Juan M. \\ FERNÁNDEZ; Marta L.' SIGNORIS y Cinthia N.'MARECO \\ demian.aq@gmail.com \\ - Tutor "Espacio DG 2019"; auxiliar de Primera en \\ Taller de Diseño Gráfico I y Tipografía I. \\ - Tutor "Espacio DG 2019"; auxiliar de Primera en Taller de \\ Diseño Gráfico l: auxiliar de Primera en Morfología I y II. \\ - Tutora "Espacio DG 2019"; auxiliar de Primera en Medios de Expresión I. \\ - Tutora "Espacio DG 2019"; jefa de Trabajos Prácticos en Taller de Diseño \\ Gráfico Il: auxiliar de Primera en Medios de Expresión I y Comunicación I y II. \\ - Tutor "Espacio DG 2019"; auxiliar de Primera en \\ Tipografía I y Taller de Diseño Gráfico II. \\ - Tutora "Espacio DG 2019"; auxiliar de Primera en \\ Taller de Diseño Gráfico l y Tipografía II. \\ - Tutora "Espacio DG 2019". \\ Diseño Gráfico, FAU-UNNE.
}

PALABRAS CLAUE

Innovación; ingresantes; aportes.

RESUMEN La presente ponencia da a conocer el análisis de las experiencias obtenidas por el grupo de tutores docentes en el cursillo de ingresantes de la carrera de Diseño Gráfico de la FAU-UNNE en el año 2019, proponiendo una mejora en las estrategias pedagógicas y didácticas, detallando veinte aportes agrupados en tres categorías como puntos de análisis: Metodología de enseñanza, Fases estructurantes y Consolidación del estudiante para con su nuevo entorno, los cuales resultaron beneficiosos para los ingresantes destinatarios de dicha propuesta, como así también para la práctica docente. 


\section{OBJETIUO}

Describir y ponderar las experiencias obtenidas en el cursillo de ingresantes "Espacio DG 2019" de la carrera de Diseño Gráfico de la FAU-UNNE al desarrollar aportes innovadores como estrategia didáctico-pedagógica por el grupo de tutores docentes.

\section{INTRODUCCIÓN}

Este artículo parte del análisis de las experiencias obtenidas por el grupo de tutores docentes en el cursillo de ingresantes de la carrera de Diseño Gráfico de la Facultad de Arquitectura y Urbanismo de la Universidad Nacional del Nordeste. Trabajar con estudiantes ingresantes en cualquier carrera universitaria es todo un desafío, considerando que la mayoría de ellos son adolescentes que egresaron recientemente de escuelas secundarias que, hoy en día, se encuentran vapuleadas debido a diversos factores sociales, económicos, entre otros. Esta etapa de la vida de los seres humanos está marcada por una multiplicidad de cambios que los atraviesan, tanto física como psicológicamente. Sumado a esto, actualmente las condiciones sociales de crisis económica, inestabilidad e inseguridad social han contribuido a aumentar la perplejidad en los jóvenes. Su mundo está vinculado por lo imprevisible, por la falta de garantías y rumbos para pensar proyectos a mediano y largo plazo, y por las limitaciones que muchas veces impone la realidad de cada uno. Este escenario desorienta a muchos jóvenes y produce una verdadera "parálisis", en la que se sienten imposibilitados para em-

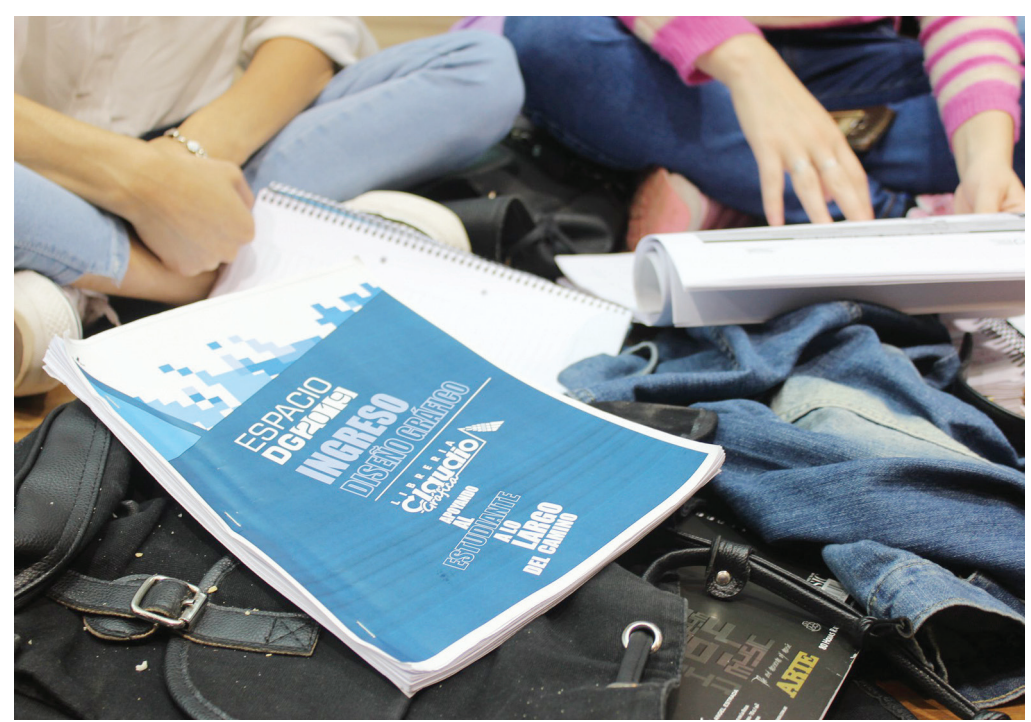

prender acciones que impliquen una decisión para sus vidas. Otro factor que considerar es que muchos de estos adolescentes provienen de distintos lugares alejados de la ciudad de Resistencia, donde se encuentran las instalaciones de la Facultad de Arquitectura y Urbanismo de la UNNE, motivo por el cual todo lo antes mencionado se magnifica dejándonos un escenario lleno de obstáculos no muy simples de sortear.

Teniendo en cuenta este contexto tan complejo fue que se pensaron las estrategias didáctico-pedagógicas para que los nuevos estudiantes puedan tener un espacio cálido donde reflexionar sobre sus futuras decisiones y conocer las características de la carrera elegida dejando atrás preconceptos o falsas expectativas. Tras el estudio de las ediciones anteriores y las devoluciones obtenidas por la comunidad académica en las diferentes jornadas de actualización y reformulación del plan de estudios de la carrera como consecuencia de los cambios sociales, culturales y tecnológicos que se están viviendo en este contexto histórico, se logró llevar a cabo una mejora en la estrategia pedagógica, con veinte aportes realizados en sus fases estructuradoras, en las metodologías de enseñanza y en propuestas que consolidaron la pertenencia del estudiante para con su nuevo entorno. Se llegó a este nuevo modelo gracias al aporte obtenido como resultado del análisis desde las perspectivas de distintos autores, que permitieron revalorizar estas metodologías en ámbitos de estudios académicos. A partir de lo expuesto, se elaboró una conclusión a modo de reflexión, a fin de dejar abierto el debate sobre la temática.

\section{DESARROLLO}

El curso del ingresante de la carrera de Diseño Gráfico de la Facultad de Arquitectura y Urbanismo de la Universidad Nacional del Nordeste, denominado "Espacio DG", es el ámbito que permite al ingresante conocer aspectos relacionados con el funcionamiento del sistema universitario en general y de la carrera en particular. Se hace foco principalmente en actividades de ambientación, relevamiento de conocimientos previos y visualización de habilidades, valores y actitudes. Se desarrollan contenidos conceptuales, procedimentales y actitudinales con el objetivo de integrar, optimizar y potenciar las competencias que los ingresantes adquirieron durante su escolarización previa. Hasta la edición 2018, el proyecto presentaba 
tres fases: una de ambientación y contenido institucional, en la cual se construyen nociones básicas de la universidady organización académica; otra de presentación y articulación de las materias del primer ciclo de la carrera y una final de conclusiones, para generar el cierre del ciclo.

A través del diálogo con estudiantes y docentes, comentarios en la comunidad académica y opiniones en reuniones acerca de la revisión y actualización del plan de estudio de la carrera, se obtuvieron valiosas devoluciones acerca de este espacio, que se convirtieron en instrumentos de evaluación y que se detallan a continuación.

\section{- El cursillo está disociado con la} carrera: la experiencia del Espacio DG se desarrolla en un clima ameno y colaborativo, ambiente que no se replica en muchas de las materias a lo largo de las cursadas.

\section{- Podría aprovecharse el espacio} para trabajar la comprensión lectora: los ingresantes presentan dificultades para interpretar consignas y analizar textos.

- Se debería instruir al grupo de alumnos a manejar ciertos aspectos básicos de los programas de diseño que necesitarán emplear desde el primer tramo de la carrera.

- Los docentes desconocen lo que se realiza en el espacio: sería pertinente dar a conocer lo que se vive en este trayecto de adaptación específico de la carrera de Diseño Gráfico.

Estos problemas mencionados no pueden ser resueltos en su totalidad en el lapso de las tres semanas en que transcurre el cursillo, ni se podrían obtener todas las soluciones de un año al otro, pero funcionaron como disparadores para comenzar a repensar las estrategias pedagógicas implementadas, reflexionar acerca de sus fortalezas y considerar las oportunidades de mejora. En la edición 2019, se implementó una propuesta de trabajo superadora, que presentó ajustes en la estrategia pedagógica, como nuevas modalidades de enseñanza, modificaciones en sus fases estructurantes y consolidación de sentido de pertenencia del estudiante para con su nuevo entorno. Para describirlas y considerarlas puntos de análisis, se las enumeró en veinte aportes, agrupados en tres categorías

\section{METODOLOGİA DE ENSEÑANZA}

La metodología deenseñanza empleada en años anteriores corresponde a clases magistrales, ejercicios individuales, grupales y actividades desarrolladas mediante la modalidad "taller". En la edición 2019, además de las convencionales, se pusieron en acción otras:

1. Cuadernillo del ingresante. La incorporación de material bibliográfico orientó las dinámicas de las tres semanas del cursillo. Acompañados de guías de lecturas y debates, los ingresantes lograron elaborar una idea más completa de lo que es el Diseño Gráfico, qué competencias deberán desarrollar a lo largo de la carrera y cuál es el mercado regional en el que se insertarán. Las lecturas ofrecidas fueron:

- ¿Qué es el diseño hoy? Comisión Asesora de la Carrera de Diseño Gráfico, "Estudio Preliminar-Bases para una evaluación del Plan de Estudios de la Carrera de Diseño Gráfico / FAU-UNNE". Pág. 1 a 7.

- Competencias Profesionales. Co- misión Asesora de la Carrera de Diseño Gráfico, "Estudio Preliminar - Bases para una evaluación del Plan de Estudios de la Carrera de Diseño Gráfico / FAU-UNNE". Pág. 8 a 10. - ¿Quétipo de diseñador es necesario? Comisión Asesora de la Carrera de Diseño Gráfico, "Estudio Preliminar Bases para una evaluación del Plan de Estudios de la Carrera de Diseño Gráfico / FAU-UNNE". Pág. 11 a 20. - Roles dentro de un grupo operativo. Trabajo recopilado y editado por Mareco, Cinthia Noelia. Espacio DG 2019. Carrera de Diseño Gráfico. UNNE. Año 2019.

- Manual Photoshop. Recuperado el 18 de enero de 2019 en http://www. professionalscat.net/manuals/pdf/ Photoshop_Basic.pdf (37 páginas). - Manual Illustrator. Recuperado el 18 de enero de 2019 en http://www. uneweb.com/tutoriales/DiplomadoDisenoDigital/Ilustrator/manual\%20 illustrator\%20cs5.pdf. (24 páginas).

\section{Dinámicas de grupos interco-} misiones. Convencionalmente, el grupo de ingresantes se divide en cinco comisiones de acuerdo con el orden alfabético, dentro de las cuales los estudiantes conforman pequeños grupos de trabajo. La interacción que se generaba bajo esta estructura los limitaba a relacionarse con los mismos compañeros durante todo el trayecto. En algunos casos presentaron disconformidad con el grupo. Comprendiendo la importancia de la dimensión social en este período de inserción a la vida universitaria, se propusieron dinámicas que permitieron a los estudiantes relacionarse e interactuar con sus compañeros de la comisión originaria y con los de las demás comisiones, formando nuevos grupos de trabajo y generando nuevos vínculos. Es 
por ello que, a lo largo de las tres semanas, se organizaron diferentes dinámicas grupales intercomisiones: - comisiones distribuidas por orden alfabético de apellidos;

- comisiones divididas en cinco colores: cian, magenta, amarillo, negro y blanco;

- comisiones divididas por animales autóctonos:

- comisiones divididas por orden alfabético de los nombres usuales.

Estas modalidades de trabajo en grupo, por un lado, sirvieron para generar más confianza en ellos mismos, en el espacio donde se estaban desenvolviendo, con los tutores, con sus compañeros; y por otro, generaron mucho entusiasmo, que se vio reflejado en la producción final de cada jornada. Esta estrategia lúdica cumplió ampliamente el objetivo previsto: "romper el hielo", facilitar a los estudiantes entablar nuevas relaciones para ir conociendo a las personas que podrían ser sus compañeros de estudio en los próximos años, ya que la carrera requiere mucho trabajo grupal. 3. Trabajo práctico. En el cursillo se solían realizar actividades diferentes en cada jornada, con poca conexión entre ellas. En la edición 2018 se optó por llevar adelante un trabajo práctico que funcionara como estructurador de los contenidos de cada clase. Esta metodología sirvió además para dar cuenta en los estudiantes de la modalidad trabajada en gran medida en todas las materias. Es por esto que en la edición 2019 se decidió replicar esta experiencia, con una nueva temática.

En una primera instancia, en grupos de aproximadamente cinco estudiantes, debían dialogar para hallar una película o serie que todos los integrantes hubiesen visto. Esta dinámica funcionó como disparador para iniciar el intercambio y reconocimiento en los pequeños grupos, a fin de "romper el hielo".
Con el avance de los días y el desarrollo de los contenidos propuestos por cada materia participante del cursillo, los grupos fueron incorporando y relacionando esos nuevos conocimientos a las ejercitaciones diarias, con el fin de elaborar -al final del cursillo- un póster integrador. Utilizar como disparador un film o serie significativo para ellos ayudó en la incorporación de los contenidos. El proceso de pensar en una serie o película elegida por ellos tuvo la intención de conocer los gustos personales de cada grupo, así como también la dinámica de exponer sus gustos y elecciones, para luego seleccionar solo una opción, hizo resaltar los roles dentro de los pequeños grupos.

\section{Muestra de las producciones fina-} les. El resultado del trabajo práctico realizado por los estudiantes fue montado en una muestra localizada en el pasillo principal de la facultad, que se expuso durante dos meses

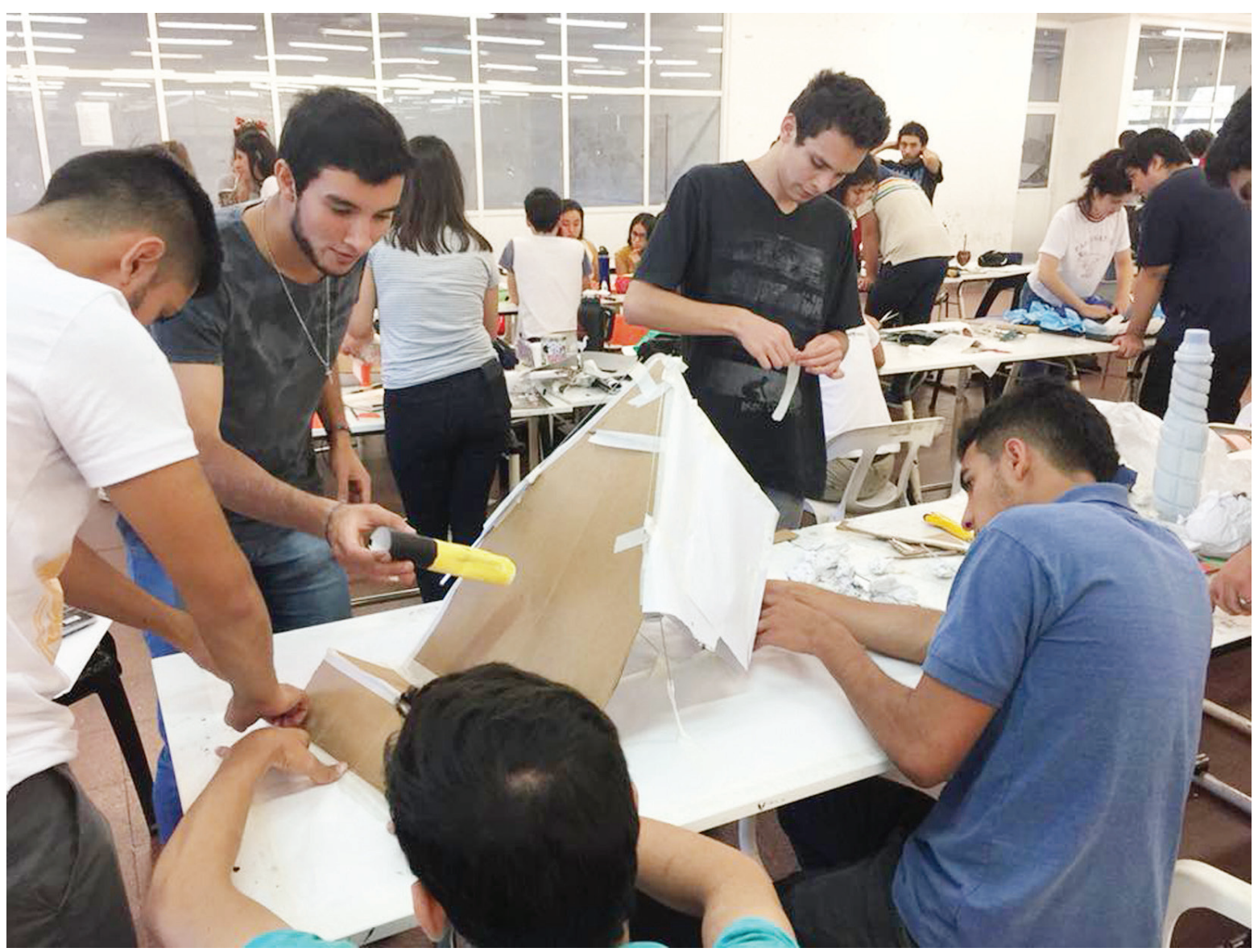


El recorrido por la muestra, como actividad de cierre del período de cursillo, resultó motivador y movilizador para los ingresantes. Ellos vieron sus pósters, resultado de semanas de trabajo, impresos junto a las producciones de sus compañeros, expuestos a toda la comunidad universitaria. Esta experiencia les generó su primera sensación de estudiantes universitarios de Diseño Gráfico, al obtener y exhibir su primer trabajo dentro de la carrera. Es notable ver la cara de satisfacción en los rostros de los estudiantes al observar expuestas sus producciones. Esta acción conlleva darle valor y reconocimiento al esfuerzo y compromiso demostrados en las semanas previas de trabajo.

5. Complementos digitales: el uso de las Tecnologías de la Información y la Comunicación (TIC) se empleaba en años anteriores con el uso de un blog, sitio de gestión de asistencias y grupo de Facebook. En esta edición 2019 detectamos nuevas necesidades, por lo cual se determinó ajustar estos dispositivos:

- Classroom (aplicación de Google): por cuestiones de tiempo, en los cierres de cada jornada no alcanzamos a visibilizar la producción que realizaban todos los grupos. Es por ello que incorporamos esta plataforma, donde los estudiantes subían las producciones diarias a fin de compartirlas con el resto de los compañeros y valorar el resultado de cada jornada.

- Grupo de Facebook: espacio para publicación de las fotos de cadajornada y socialización de avisos, recordatorios y novedades de modo oficial.

- Cinco grupos de WhatsApp, uno por cada comisión: para la coordinación de información espontánea, avisos, recordatorios y novedades. En algunas comisiones, limitaciones de horarios para dialogar y normas explícitas de respeto ayudaron a los estudiantes a desenvolver cuestiones actitudinales.

Estos espacios complementarios funcionaron como apoyatura al

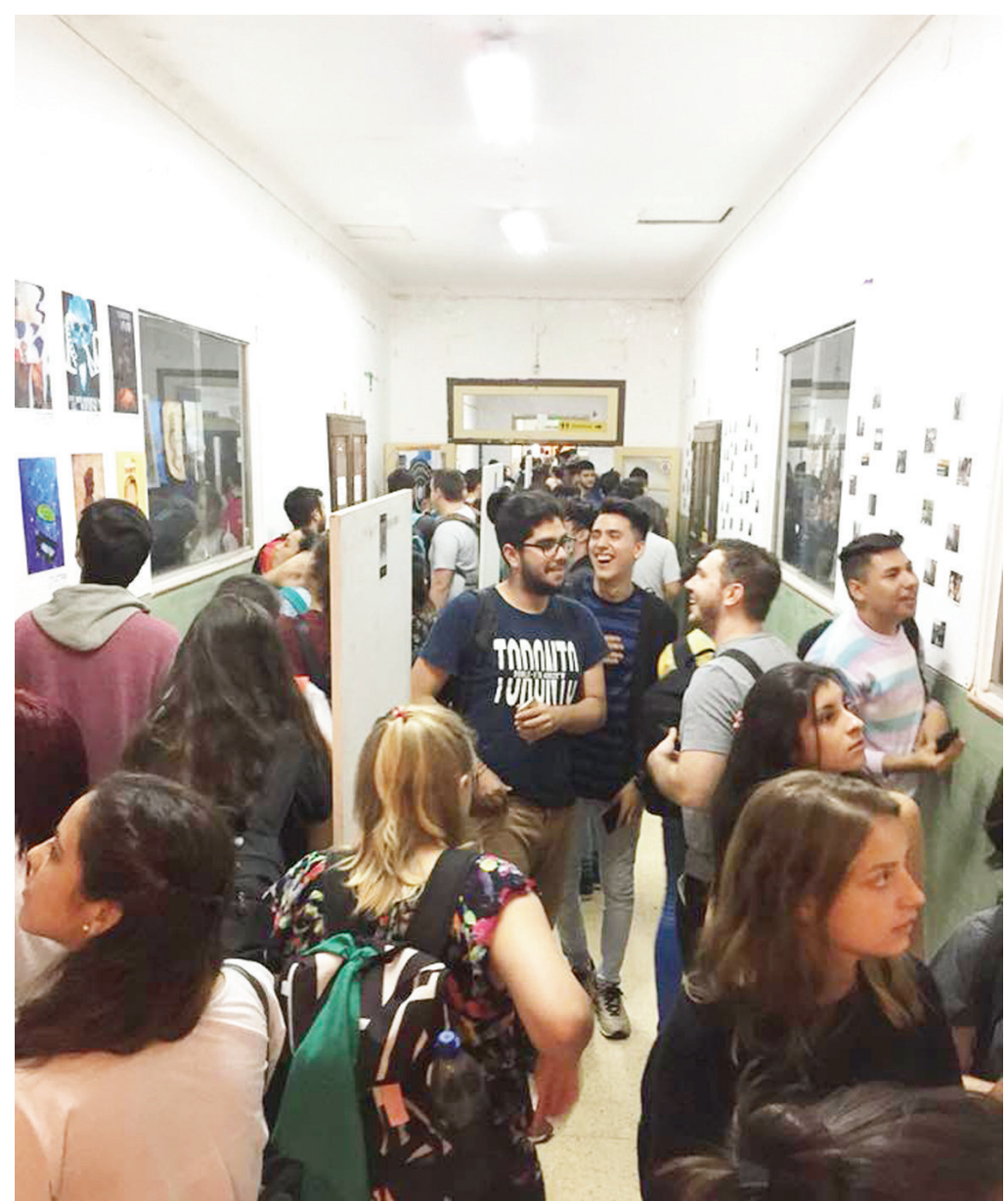

trabajo presencial, y fueron regulados o moderados por los tutores, a fin de que no se desvirtuaran los propósitos específicos de cada uno.

6. Proyección de videos: continuando con los beneficios de las TIC, se proyectaron videos con contenidos conceptuales, emocionales y musicales, seleccionados por los tutores pares, con el fin de estimular la llegada a clases y generar temas de charlas entre los estudiantes mientras se esperaba el inicio de las actividades.

\section{FASES}

\section{ESTRUCTURANTES}

Se realizaron ajustes y se incorporaron nuevos espacios a las tres fases estructuradoras del cursillo mencionadas anteriormente.
7. Espacio Tecnológico. La cuestión tecnológica no se aborda como contenido, por cuestiones del plan de estudios, hasta en años avanzados de la carrera, por lo que el acercamiento a los programas de diseño y la orientación respecto de qué ordenador les resultará útil depende generalmente de la iniciativa del estudiante y de los consejos que sea capaz de pedir. Es por ello que se planteó como objetivo introducirlos en los conocimientos básicos de los programas y orientarlos respecto de cómo organizarse para equiparse de manera inteligente. De esta manera, organizamos las siguientes ponencias: - Introducción a software de diseño: mapa de bits.

- Introducción a software de diseño: vectores.

- Tips para la elección de ordenadores.

8. Presentaciones de las materias del primer ciclo de la carrera. Esta 
modalidad se realiza desde años anteriores, y son expuestas por los adjuntos a cargo de las asignaturas del primer nivel. En la edición 2019, se contó con la participación activa de tutores que integran dichas cátedras y que fueron responsables de los cierres de las actividades de cada la jornada.

- Taller de Diseño Gráfico 1. Adjunta: María Bernabela Pelli. Cierre: Damián Aquino.

- Comunicación 1 e Historia 1.Adjuntos: Adriana Echeverría y Miguel Riera, respectivamente. Cierre: Cintia Slobodiuk y Luján Signoris.

- Morfología 1.Adjunta: Susana Geat. Cierre: Fernando Estévez.

- Medios de Representación 1. Adjunta: Daniela Salinas. Cierre: Paula Pettinichi.

- Tipografía 1. Adjunto: Tomás Ricchieri. Actividad intermedia: Juan Manuel Fernández.

En este espacio, los ingresantes despejaron algunas dudas respecto de las cursadas, regularidades y aprobación de las materias, además de elaborar una idea general del contenido de estas. La actividad de cada jornada estuvo planificada de manera tal que los conceptos básicos que incorporaron de estas presentaciones pudieran ser aplicados a la producción de cada grupo.

9. Jornadas de exposiciones de diseñadores gráficos profesionales. Transitando la tercera y última semana del cursillo, los ingresantes conocieron diseñadores que, en el ejercicio de su profesión, fueron especializándose en distintas ramas del diseño; trabajando con equipos interdisciplinarios, orientándose a la investigación, a las instituciones públicas, al diseño tipográfico al diseño multimedial, a la ilustración y a los emprendimientos. En cada una de estas charlas, el panorama se fue ampliando, y cada ingresante se interesó por una u otra orientación disciplinar o metodología de trabajo. Conocer referentes reales, que se desarrollan satisfactoriamente en el mercado laboral regional, les resultó muy inspirador, además de permitirles evacuar dudas respecto de la salida laboral que ofrece esta profesión. Las ponencias fueron realizadas por los siguientes profesionales: - Aníbal Pauttazo: ¿Por qué estudiar Diseño Gráfico? Algunos mitos y verdades.

- Ludmila Strycek: ¿Qué come - de qué se alimenta - el Diseño?

- Enrique Aris Rousselot: Desdibujar el Ego.

- Pilar Polo: Mi proceso hacia la Ilustración

- Katya Bradford: El impacto social del Diseño (Diseño Gráfico en Organismos Públicos).

- Ramiro Núñez Rolón y Eugenia Kusevitzky: Diseño y Activismo Gráfico.

- Damián Calderón: De estudiante a Diseñador de Apps

10. Jornadas de exposiciones de estudiantes de la carrera. Esta jornada fue muy significativa, ya que los mismos estudiantes contaron sus experiencias, en relación con la carrera y la vida universitaria en general. Desde su lugar de pares,

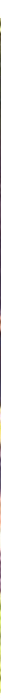


compartieron sus aprendizajes y los consejos que consideraron pertinentes. También expusieron una selección de sus trabajos prácticos, mostrando no solo el resultado, sino también los procesos y dificultades que tuvieron que resolver. Las charlas fueron realizadas por las siguientes personas:

- Estudiantes de 2. ${ }^{\circ}$ ciclo: Ana Medina, Franco Kucinskas y Uriel Insaurralde. - Estudiante de $3 .^{\circ}$ ciclo: Ana Soler. - Estudiantes de $4 .^{\circ}$ ciclo: Agustín Gómez, Nuria Pedrozo y Sabrina Giusiano.

11. Jornada integradora. En ella las comisiones de ingresantes debían realizar una producción final del espacio de manera libre y colaborativa, realizando una síntesis de los aprendizajes obtenidos y las experiencias compartidas. En los resultados se reflejaron el entusiasmo y la creatividad puestos en la actividad, que resultaron en dramatizaciones, producciones visuales, intervenidas por todo el grupo, y la realización de videos

12. Jornada de camaradería. En la última jornada del cursillo, los ingresantes realizaron una recorrida por los diferentes sectores del campus UNNE, como las bibliotecas, el comedor, las diferentes facultades y espacios verdes, guiados por los tutores. Luego participaron de actividades recreativas y compartieron un almuerzo con choripanes en el club CUNE. Este encuentro generó lazos amenos entre estudiantes y tutores y un cierre exitoso. Fue un momento muy significativo, ya que tuvieron la oportunidad de re-conocerse en un ámbito no académico, lo que les permitió afianzar las nacientes relaciones.
Consolidando la pertenencia del estudiante para con su nuevo entorno En la edición 2019 también se llevaron a cabo acciones que pretenden dar valor y potenciar este espacio dentro y fuera de la institución.

\section{Registro fotográfico de cada} jornada. Fueron compartidos diariamente en el grupo de Facebook "Espacio DG 2019". Los estudiantes buscaban ser capturados para compartir el material en sus redes.

14. Agradecimientos, reflexiones finales, palabras de ingresantes y entrega de certificados a los tutores y tutores pares por haber participado en las tres semanas de duración del cursillo. Este espacio generó un intercambio de opiniones, vivencias y resultados de las tres semanas, en el cual se pudo realizar una reflexión conjunta del proceso realizado entre todos los involucrados en esta experiencia.

15. Gestión con empresa del medio para facilitar el acceso al cuadernillo del ingresante (50\% de descuento) y realizar su primera impresión digital de un trabajo práctico (100 $\%$ bonificada), para asegurar una muestra final de la cursada de alta calidad que también sea un recuerdo para cada estudiante, teniendo en cuenta el contexto económico en el que estamos insertos.

16. Institucionalización gráfica del espacio por medio de la presencia de banners que fueron logrados por financiamiento compartido con imprenta del medio, que a su vez propició diez vouchers para impresiones a los estudiantes. Los banners sirvieron para dar valor al espacio, y los vouchers, como estímulos para el inicio del cursado.
17. Trascendencia de la producción diaria por medio de publicaciones en canales oficiales, como la cuenta de Instagram "sae.fau", perteneciente a la Secretaría de Asuntos Estudiantiles de la FAU-UNNE, compartiendo una publicación diaria de diez fotos significativas de cada jornada y "stories" de las actividades en vivo y algunas producciones de cada día. Esta acción repercute significativamente en la autoestima de los estudiantes, ya que ven sus producciones exhibidas para otras personas ajenas al cursillo de ingreso y al ámbito universitario. Esta herramienta también propicia compartir los hechos sucedidos en el espacio a otras personas que no pertenezcan a él, como estudiantes de otros niveles, de otras carreras, personal de la institución y público en general.

18. Sistema de Identidad Visual del Espacio DG 2019, que tiene las siguientes piezas de comunicación: - banners:

- portada del cuadernillo;

- proyecciones;

- portada en las plataformas digitales; - certificados:

- invitaciones a las jornadas de los profesionales y estudiantes.

Estas piezas gráficas son claves para que los alumnos reconozcan la importancia de la identidad como construcción de sentimientos de pertenencia, sentimiento que pretendemos inculcar a todos los ingresantes para que se consideren parte de la institución.

19. Formulario de evaluación final creado con la plataforma Google Forms, con el objetivo de que los estudiantes realicen una evaluación crítica de las experiencias vividas 


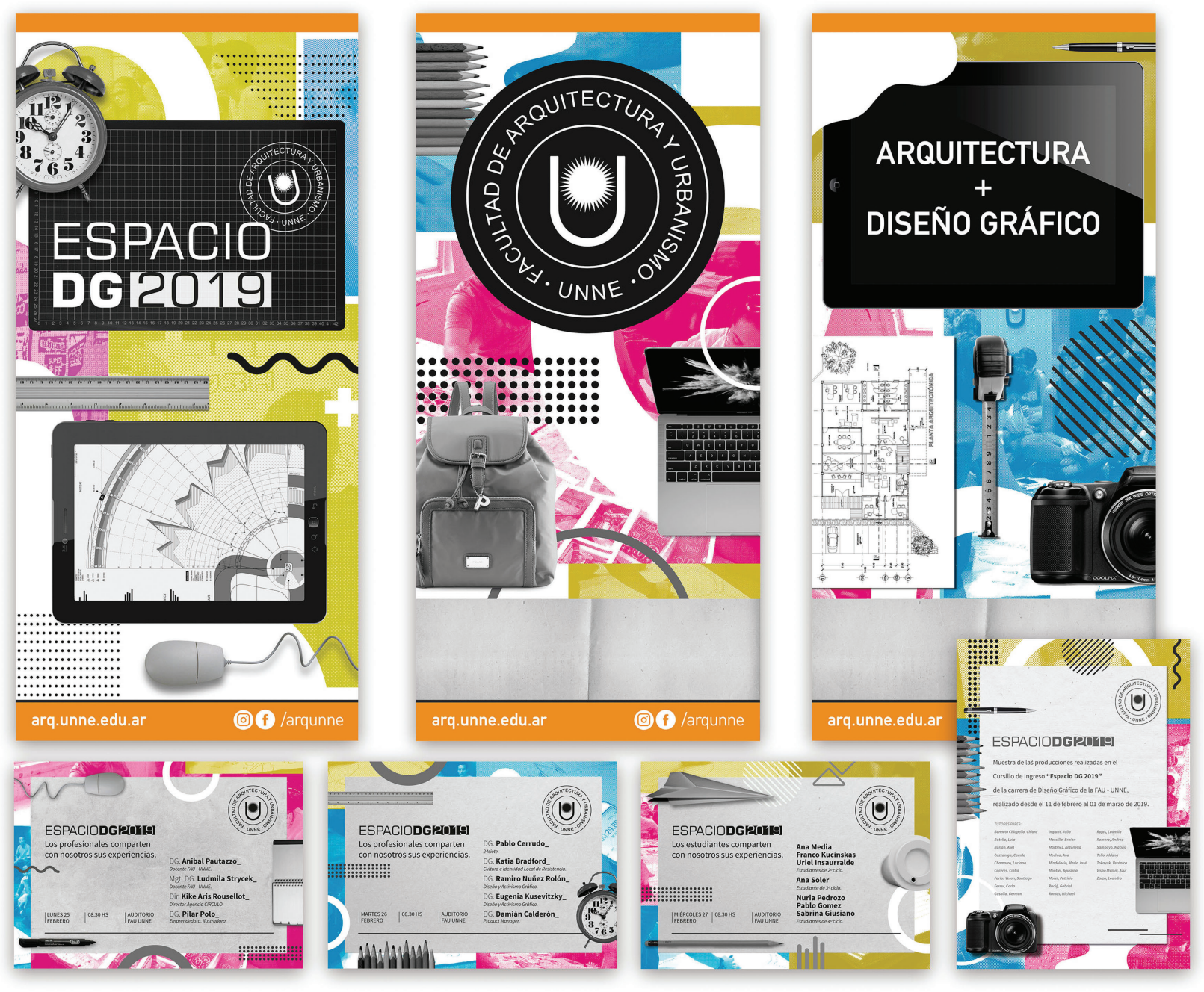

en el cursillo. Los interrogantes planteados fueron pensados para que los alumnos reflexionen sobre su propia práctica y también puedan expresar su punto de vista sobre la metodología empleada en el espacio. Su objetivo es ser insumo e instrumento de evaluación para realizar futuras observaciones y mejoras en las siguientes ediciones.

20. Abrir el Espacio. Pensando en que la experiencia vivida en este ciclo no terminó al finalizar las tres semanas de duración, se apuesta a abrir la realidad de lo que se vive en el cursillo a nuevos ámbitos académicos de intercambio y reflexión, como una práctica saludable para dar cuenta de las decisiones que se han tomado, manifestando los resultados. Para dar inicio a este punto, el cuerpo de docentes a cargo del cursillo decidió exponer las experiencias obtenidas mediante la participación de diversos congresos y jornadas institucionales que permitieran adquirir e intercambiar experiencias similares y fomentar el florecimiento de nuevos aportes en estrategias pedagógicas que contribuyan a mejorar la práctica docente y potenciar las dinámicas de las futuras ediciones.

\section{REFLEXIONES CON APORTES TEÓRICOS}

Las nuevas propuestas en las metodologías de enseñanza, en las fases estructurantes y en las acciones llevadas a cabo para la consolidación del sentido de pertenencia en los estudiantes para con el cursillo de ingreso, permiten reflexionar sobre estrategias que implican nuevas formas de producción de conocimiento, nuevos modos de aprender, de interactuar, transformaciones integrales, procesuales, complejas. Estas experiencias posibilitan considerar que en el cursillo de ingreso se logró una innovación en cuanto a su estrategia pedagógica. Una experiencia innovadora que se define por dos rasgos esenciales: la ruptura con el estilo didáctico habitual y el protagonismo de los actores involucrados en ella. Según Elisa Lucarelli, "la innovación como ruptura implica entenderla como la interrupción de una determinada forma de comportamiento que se repite en el tiempo y se legitima" (Lucarelli, 2009, p. 52). De esta forma, 
las innovaciones involucran siempre creatividad, procesos producidos de manera original para su contexto de realización, y estas producciones son llevadas a cabo por sujetos y grupos, lo cual nos remite al protagonismo de sus interventores. Las innovaciones "son protagónicas en el sentido de que sus creadores (...) son los personajes principales de la acción y toman parte en los momentos significativos de la misma" (Lucarelli, 2009, p. 56).

Dicho de otro modo, la innovación implica protagonismo y que los sujetos tengan la posibilidad de decir su palabra, habilitando así nuevas configuraciones de poder (Leite, Genro y Braga, 1997, citados en Lucarelli, 2009). Para ello, la participación y el compromiso de los sujetos in- volucrados son necesarios, lo cual requiere la subversión consciente de las relaciones de poder establecidas hasta el momento. Este involucramiento y participación del estudiante implica un aprendizaje significativo, ya que el aprendiz relaciona la nueva información con sus conocimientos y experiencias previas, construye significado, da sentido a lo aprendido y entiende su ámbito de aplicación y relevancia en situaciones académicas y cotidianas (Díaz Barriga, 2003)

Las Tecnologías de la Información y la Comunicación (TIC) también obtuvieron su análisis y fueron utilizadas para satisfacer algunas necesidades requeridas en cuestiones didácticas, como por ejemplo elaborar un espacio en donde todos los estudiantes puedan observar las producciones de sus pares. Esto nos llevó a pensar que se necesitaba implementar un cambio metodológico acorde con las necesidades presentes.

Se puede usar la tecnología para hacer las mismas actividades que siempre... Sin embargo, consideramos que el objetivo no es usar tecnología sino adaptar la educación a las necesidades actuales y, por tanto, se precisa un cambio metodológico

La apropiación de la tecnología pasa por el uso personalizado, pero también por el trabajo colaborativo, la negociación, el trabajo con estudiantes y profesores más allá del propio centro y de la propia aula, el desarrollo de competencias comunicativas, de diseño y de creación de materiales. No se trata sólo de consultar información sino también de crear nuevos materiales y conocimientos (Gros Salvat, 2004, p.7).
El pedagogo francés Philippe Meirieu señala que la dificultad de la educación es que

la instrucción es obligatoria, pero no tenemos poder sobre la decisión de aprender. Esta no es producto de ninguna 'causa' mecánica, no se deduce de ningún modo de ser hipotético, no puede pronosticarse a partir de ningún análisis a priori. La decisión de aprender cada cual la adopta solo, por razones que, sin embargo, no son propias de quien las adopta (Meirieu, 1998, p. 79).

Es por esto que consideramos esencial acompañar a los alumnos para que puedan darse las condiciones y ellos tomen las riendas de su proceso de aprendizaje, ya que por más que los profesores pongan todo su empeño y dedicación en enseñar, el aprendizaje se da solo si el sujeto lo permite. Es un proceso personal e intransferible. Nuestra tarea como docentes es crear situaciones de aprendizaje "amigables" teniendo en cuenta las situaciones y contextos a los que nos enfrentamos. Para esto es necesario conocer a nuestros estudiantes de manera holística.

La estrategia planteada en el presente artículo pretende reestructurar todos los aspectos de la educación: la vida universitaria, los contenidos de las materias, el rol del profesional, de los estudiantes y de los docentes, la manera de enfocar el proceso de aprendizaje, la importancia de las capacidades, valores y sentimientos frente a las nuevos desafíos, las nuevas tecnologías, y que la educación nos provea un marco integral y coherente que incluya todos los aspectos que deben ser considerados en una propuesta educativa innovadora. 


\section{CONCLUSIÓN}

Tomando como ejes orientadores las premisas a las que arribamos en las consultas, escuchas y análisis retrospectivo del cursillo, concluimos en que se ha logrado una mejora sustancial de las actividades respecto de las necesidades específicas de los ingresantes a esta carrera, una innovación en la estrategia didáctico-pedagógica. Luego de revisar lo vivenciado en el "Espacio DG 2019", podemos exponer que los veinte aportes realizados ayudaron a paliar diversas problemáticas que los ingresantes venían cargando. La desorientación y las crisis de enfrentarse a situaciones desconocidas, la incertidumbre sobre los planes de estudios y la vida universitaria, el desarraigo, el reto de entablar nuevas relaciones personales con personas diferentes de las que están acostumbrados a tratar, entre otros tantos factores negativos con los que se enfrentan los estudiantes ingresantes de la carrera de Diseño Gráfico-como seguramente sucede en otras carreras universitariaspueden verse atenuados con la guía de tutores que orienten y aconsejen a los nuevos alumnos no solo en el ámbito académico, sino también en lo personal y social (áreas que son trabajadas en el recorrido del listado de puntos citados).

Estimamos que los aportes propuestos en el presente artículo pueden ser replicados, parcial o totalmente, en otras carreras de nuestra casa de estudios, en otras instituciones, e incluso en otro país con situaciones culturales, sociales y económicas similares a la nuestra, ya que contribuyen al mejoramiento de la calidad de la enseñanza, generan una mayor predisposición para el aprendizaje, una disminución de la deserción y el desgranamiento en los primeros meses de cursado, y todo esto influye en la imagen positiva de la universidad.

\section{BIBLIOGRAFIA}

DÍAZ BARRIGA ARCEO, F. (2003). "Cognición situada y estrategias para el aprendizaje significativo". En Revista electrónica de investigación educativa, vol. 5, N. ${ }^{\circ}$ 2. Recuperado de http://redie.uabc.mx

LUCARELLI, E. (2009). Teoría y Práctica en la Universidad. La innovación en las Aulas. Buenos Aires: Miño y Dávila.

MEIRIEU, P. (1998) Frankenstein educador. Barcelona: Laertes Pedagogía.

GROS SALVAT, B. (2004). De cómo la tecnología no logra integrarse en la escuela a menos que... cambie la escuela. Barcelona, Universidad Autónoma de Barcelona, Jornada 2004, Espiral. 